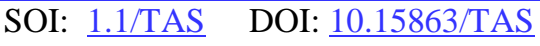 International Scientific Journal Theoretical \& Applied Science}

p-ISSN: 2308-4944 (print) e-ISSN: 2409-0085 (online)

Year: $2015 \quad$ Issue: 03 Volume: 23

Published: $30.03 .2015 \quad$ http://T-Science.org
Sergey Aleksandrovich Vorobyev

Candidate of Technical Sciences, associate professor of Don State Technical University, Russia v.serge.79@mail.ru

Sergey Sergeevich Vorobyev

Candidate of Technical Sciences, associate professor of Don State Technical University, Russia

Andrey Stanislavovich Reshenkin

Candidate of Technical Sciences, head of department of Don State Technical University, Russia

Roman Aleksandrovich Goncharov

Candidate of Technical Sciences, associate professor of Don State Technical University, Russia

Alexander Grigoryevich Tihomirov

Candidate of Technical Sciences. associate professor of Don State Technical University, Russia

\title{
NECESSARY CONDITIONS FOR OPTIMAL DAMPING SUSPENSION CAR WITH DYNAMIC ACTION
}

Abstract: There are necessary conditions for optimal damping of oscillators in suspension design of the vehicle with dynamic action in this article. Received theoretical position were used to develop a methodology for calculating the dynamic characteristics of vehicles with optimal vibration damping systems, which is designed to calculate the vertical angular oscillations of biaxial the vehicle in motion.

Key words: damping, oscillations, method, dynamic characteristics, transport.

Language: Russian

Citation: Vorobyev SA, Vorobyev SS, Reshenkin AS, Goncharov RA, Tihomirov AG (2015) NECESSARY CONDITIONS FOR OPTIMAL DAMPING SUSPENSION CAR WITH DYNAMIC ACTION. ISJ Theoretical \& Applied Science 03 (23): 97-100.

Soi: http://s-o-i.org/1.1/TAS*03(23)18 Doi: crossef http://dx.doi.org/10.15863/TAS.2015.03.23.18

\section{НЕОБХОДИМЫЕ УСЛОВИЯ ОПТИМАЛЬНОГО ДЕМПФИРОВАНИЯ КОЛЕБАНИЙ ПОДВЕСКИ АВТОМОБИЛЯ ПРИ ДИНАМИЧЕСКОМ ВОЗДЕЙСТВИИ}

Аннотация: B статье сформулированы необходимые условия оптимального демпфирования колебаний конструкиий подвески автотранспортного средства при динамическом воздействии. Полученные теоретические положения применены для разработки методики расчета динамических характеристик автотранспортных средств с системами оптимального демпфирования колебаний, которая предназначена для расчета вертикально-угловых колебаний двухосного автотранспортного средства в движении.

Ключевые слова: демпфирование, колебания, методика, динамические характеристики, транспорт.

В настоящее время адаптивные подвески автомобилей получают все более широкое распространение (как правило, в автомобилях премиум-класса). В адаптивных (или активных) подвесках степень демпфирования изменяется автоматически в зависимости от состояния дорожного полотна, параметров движения и принудительно водителем в зависимости от его предпочтений [1].

Альтернативой автоматической адаптивной подвеске является винтовая подвеска (колойверы). Колойверы позволяют механически сжимать пружину, (при помощи резьбовых регуляторов) относительно амортизатора, регулируя, таким образом, его жестокость.
Амортизатор в такой подвеске также может быть выполнен с регулятором жесткости. Колойверы широко применяются в автоспорте.

И в том, и в другом случае оптимальные настройки жесткости подвески автотранспортного средства позволяют свести к минимуму амплитуду и время колебательных процессов в подвеске. Однако такие системы подвески доступны не всем транспортным средствам, поэтому задача определения условий оптимального демпфирования колебаний традиционных типов подвесок остается актуальной.

При решении научных задач в качестве физических моделей объектов исследования 
принимаются системы с сосредоточенными параметрами, представляющие собой совокупность абсолютно твердых тел, соединенных друг с другом и основанием упругим подвесом [2-4]. Их математическими моделями являются системы обыкновенных дифференциальных уравнений, полученных в обобщенных координатах на основе интегрального принципа ГамильтонаОстроградского или дифференциального принципа Лагранжа второго рода. Вывод условий оптимальности осуществляется методом принципа максимума Понтрягина [5-8].

Под оптимальными понимаются такие алгоритмы управления жесткостью, при которых энергия колебательного движения снижается до заданной величины за минимальное время. Задача формулируется так:

Для колебательного процесса, описываемого системой уравнений (1) при начальных условиях (2) определить алгоритм управления матрицей квазиупругих коэффициентов, чтобы изменение кинетической энергии колебательного движения от начального значения $T_{(0)}$ до заданного конечного $T_{(k)}$ происходило за минимальное время (3). При этом на коэффициенты жесткости могут накладываться ограничения типа неравенства (4).

$$
\begin{gathered}
A \ddot{z}+C z=q(t, C) \\
t=0, \quad z=z_{(0)}, \quad \dot{z}=\dot{z}_{(0)}, T_{0}=\frac{1}{2} \dot{z}_{(0)}^{T} A \dot{z}_{(0)} \\
t=t_{(k)}, \quad T_{(k)}=\frac{1}{2}\left(\dot{z}_{(k)}^{T} A \dot{z}_{(k)}\right) ; \\
J=\int_{0}^{t_{(k)}} d t \rightarrow \min ; \\
c_{i k} \in\left\lfloor\left(c_{i k}\right)_{\min ,}\left(c_{i k}\right)_{\max }\right\rfloor .
\end{gathered}
$$

Здесь $\mathrm{Z}, \dot{\mathrm{Z}}$ - векторы обобщенных координат и обобщенных скоростей, размерностью $k \times 1$ каждый; $A$ - матрица коэффициентов инерции размерностью $\mathrm{n} \times \mathrm{n}$; С- матрица квазиупругих коэффициентов $n \times n ; \quad q(t, C) \quad$ - вектор обобщенной силы размерностью $n \times 1 ; T-$ кинетическая энергия колебательного движения системы; $(o)$, $(k)$ индексы, означающие начальное и конечное состояния.

Такая формулировка задачи обусловлена тем, что кинетическая энергия является квадратичной формой обобщенных скоростей и характеризует интенсивность колебательного, динамического процесса. Скорость ее убыли будет характеризовать эффективность гашения колебаний и, естественно, за минимальное время конструкция будет испытывать меньшее число толчков и знакопеременных нагружений.

Для определения условий оптимальности закона изменения жесткости использовалось понятие игольчатой вариации и стандартное расширение фазового вектора $z(t)$ путем переобозначения функционала $J$ и времени $t$ :

$$
\begin{gathered}
z=\left(z^{0}, z^{1}, \ldots, z^{n}, z^{n+1}\right) ; \\
z^{0} \equiv J, \quad \dot{z}^{0}=1 ; z^{n+1} \equiv t, \quad \dot{z}^{n+1}=1
\end{gathered}
$$

Путем введения $n+2$ - мерного вектора сопряженных

$\psi=\left(\psi_{0}, \psi_{1}, \ldots, \psi_{n}, \psi_{n+1}\right)^{T}$ для фазовых

координат и множителя Лагранжа $\lambda$ для краевого условия $t=t_{(\kappa)}$ вместо исходного функционала строился расширенный функционал по правилу

$$
\begin{aligned}
J^{*}=\int_{0}^{t_{k}}\left\{\begin{array}{l}
\left(\dot{z}^{0}-1\right) \psi_{0}+ \\
\left.\sum_{i, j=1}^{n} \psi_{i}\left[A_{i j} \ddot{z}^{j}+c_{i j} z^{j}-q_{i}\left(Z^{n+1}, C\right)\right]\right\} d t+ \\
+\left(\dot{z}^{n+1}-1\right) \psi_{n+1}
\end{array}\right] \\
+\lambda\left(T_{(k)}-T_{(0)}\right)= \\
=\int_{0}^{t_{k}} F(z, \psi, C) d t+\lambda\left(T_{(k)}-T_{(0)}\right)
\end{aligned}
$$

где $F(z, \psi, C)$ - подынтегральная функция.

$$
\begin{gathered}
\delta C=C_{\varepsilon} \backslash \tilde{C} ; \\
C_{\varepsilon}(t)=\left\{\begin{array}{ll}
C(t), & t \in[\tau, \tau+\varepsilon l] ; \\
\widetilde{C}(t), & t \notin[\tau, \tau+\varepsilon l] ;
\end{array} \quad \varepsilon\langle\langle 1,\right.
\end{gathered}
$$

Выражение (7) позволяет определить главную часть приращения расширенного функционала.

Для этого расширенный функционал можно представить в виде суммы трех интегралов на временных интервалах

$$
\begin{aligned}
& t \in[0, \tau], \quad t \in[\tau, \tau+\varepsilon l] ; \\
& t \in\left[\tau+\varepsilon l, t_{(k)}\right] \\
& J_{\varepsilon}^{*}=\int_{0}^{\tau} F_{\varepsilon} d t+\int_{\tau}^{\tau+\varepsilon l} F_{\varepsilon} d t+ \\
& \int_{\tau+\varepsilon l}^{t_{(k)}} F_{\varepsilon} d t+\lambda\left(T_{\varepsilon(k)}-T_{0}\right)
\end{aligned}
$$




$$
\begin{aligned}
& \widetilde{J}^{*}=\int_{0}^{\tau} \widetilde{F} d t+\int_{\tau}^{\tau+\varepsilon l} \widetilde{F} d t+ \\
& \int_{\tau+\varepsilon l}^{t_{(k)}} \widetilde{F} d t+\lambda\left(\widetilde{T}_{(k)}-T_{(0)}\right)
\end{aligned}
$$

Тогда главная часть приращения функционала равна разности между варьированным и неварьированным значениями расширенного функционала

$$
\begin{aligned}
& \left.\Delta J=J_{\varepsilon}^{*}-\tilde{J}^{*}=\varepsilon l \mid F\left(z, C_{\varepsilon}, \psi\right)-F(z, \tilde{C}, \psi)\right]+ \\
& +\int_{\tau+\varepsilon l}^{t_{(k)}}\left[F\left(z_{\varepsilon}, \tilde{C}, \psi\right)-F(\tilde{z}, \tilde{C}, \psi)\right] d t+\lambda\left(T_{\varepsilon(k)}-\tilde{T}_{(k)}\right) .
\end{aligned}
$$

Учитывая, что на первом интервале $t \in[0, \tau]$ управления неварьированы и поэтому интегралы одинаковы; на втором интервале $t \in[\tau, \tau+\varepsilon l] \quad$ приращение функционала получается только за счет игольчатой вариации управления (жесткости); на третьем интервале $t \in\left\lfloor\tau+\varepsilon l, t_{(k)}\right\rfloor$ управления не варьированы, а приращения функционала получается за счет вариаций обобщенных координат. Тогда после соответствующих преобразований получим необходимые и достаточные условия оптимальности в форме теоремы 1 принципа максимума.

Tеорема 1. Для того, чтобы управления $\mathrm{C}(t)$ и соответствующие им обобщенные координаты $\mathrm{z}(t)$ доставляли минимум функционалу быстродействия при уменьшении кинетической энергии до заданного конечного значения, необходимо и достаточно существование ненулевой непрерывной вектор-функций $\psi(t)$, удовлетворяющей сопряженной системе уравнений

$$
\begin{gathered}
\dot{\psi}_{0}=0 ; \dot{\psi}_{n+1}=-\sum_{i=1}^{n} \psi_{i} \dot{q}_{i} \\
\sum_{i=1}^{n}\left(\ddot{\psi}_{i} A_{i j}+\psi_{i} c_{i j}\right)=0, \quad j=\overline{1, n}
\end{gathered}
$$

и условиям трансверсальности в конечный момент времени

$$
\begin{aligned}
& t=t_{(k)}, \quad \psi_{0}=1 ; \quad \psi_{i(k)}=-\lambda \dot{z}_{(k)}^{i}, \\
& \psi_{n+1}=0, \quad i=\overline{1, n}
\end{aligned}
$$

а функция Гамильтона

$$
\begin{aligned}
& H(z, \psi, \stackrel{\circ}{C})= \\
& \sum_{i, j=1}^{n} \psi_{i}\left[c_{i j} \dot{z}^{j}-q_{i}(C)\right]=\max _{C \in \bar{C}} H(\psi, t, C)
\end{aligned}
$$

при любом $t \in\left[\mathbf{O}, \boldsymbol{t}_{(\boldsymbol{k})}\right]$ достигала своего максимального значения по всем $C \in \bar{C}$.

Второе положение позволяет обосновать возможность переноса краевых условий в конечный момент времени в любую точку интервала времени и формулируется теоремой 2: Если $C(t)$ - оптимальное управление, изменяющее за данный промежуток времени $\tau_{1}>0$ кинетическую энергию системы на максимальную величину $T\left(\tau_{1}\right)-T_{(0)}=T_{1 \max }$, то $C(t)=\stackrel{\circ}{C}(t) \quad$ есть оптимальное по быстродействию управление задачи демпфирования колебаний системы.

Tеорема 3: Оптимальное по быстродействию управление удовлетворяет принципу максимума:

$$
\max H(z, \psi, C) \rightarrow \max _{C \in \bar{C}} H(z, \dot{z}, \lambda, C)
$$

Полученный результат принципиально облегчает проблему расчета оптимальных алгоритмов управления жесткостью, т.к. в этом случае закон управления формируется по текущему значению обобщенных координат. С учетом доказанного, уравнение для определения оптимальных коэффициентов жесткости $\mathrm{c}_{\mathrm{ij}}$ амортизаторов представляется в виде:

$$
c_{i j}=\left\{\begin{array}{l}
c_{i j \max }, \operatorname{signiz}^{i}\left(z^{i}-q_{i}\right)=+1 \\
c_{i j \min }, \operatorname{signiz}^{i}\left(z^{i}-q_{i}\right)=-1 .
\end{array}\right.
$$

Теорема 4. Для того, чтобы постоянные параметры $\mathrm{p} \in \mathrm{C} \bigcup \mathrm{K}$ и соответствующие им обобщенные координаты z доставляли минимум функционалу точности (14), необходимо и достаточно существование непрерывной векторфункции $\lambda(t)$, удовлетворяющий сопряженной системе уравнений $\quad \dot{\lambda}+\mu(z-y)^{T} \Gamma=0 \quad$ и нулевым условиям трансверсальности $t=t_{(k)}, \quad \lambda=0$, и функция Гамильтона $H=-\lambda u+\frac{1}{2} \mu u^{T} E u=\max _{u \in \bar{R}} H(\lambda, u)$, при любом $t \in\left[0, t_{(k)}\right\rfloor$ достигала своего максимального значения. При этом функции 
чувствительности

дифференциальным

чувствительности.

После несложных математических преобразований получаются расчетные уравнения для параметров системы, решение которых сводится к постоянной величине,

$$
\begin{gathered}
\dot{p}+\mu S(z-y)^{T} T=0, \\
\dot{S}+\mu S^{T} T S=0, \\
t=0, \quad p=0, \quad S=S_{(0)}, \quad t \rightarrow \infty, \\
S \rightarrow 0, p \rightarrow \text { const }
\end{gathered}
$$

где $S=\frac{\partial p}{\partial \lambda} \quad-$ матрица чувствительности параметров к сопряженным переменным, причем $S=S^{T}$ - симметричная матрица.

Полученные теоретические положения были применены для разработки методики расчета динамических характеристик объектов с системами оптимального демпфирования колебаний [9-10], для вертикально-угловых колебаний двухосного транспортного средства при движении по неровной дороге.

\section{References:}

1. Kuchvid RP (2001) Ispitaniya avtomobilya:uchebnik- Moscow: MGIU, 2001.$351 \mathrm{p}$.

2. Kostoglotov AI, Shevcova LA (1987) Optimalnoe vozbuzhdenie rezonansnykhkolebanij uprugikh system prikladnaya mekhanika, 1987, T.23 №6, pp.23-30.

3. Kovaleva AS (1990) Upravlenie kolebatelnymi i vibroudarnymi sistemami. Moscow, -Nauka1990. $-256 \mathrm{p}$.

4. Natshif A, Dzhouns D, Khenderson D (1988) Dempfirovanie kolebanij. Moscow, -Mir, 1988. -448 p.

5. Bishop R (1986) Kolebaniya /per s angl. pod red.-Ya.G Panovko.-3-e-izd, -Moscow, -Nauka1986. $-190 \mathrm{p}$.

6. Komkov V (1975) Teoriya optimalnogo upravleniya dempfirovaniem kolebanij prostykh-uprugikh sistem, -Moscow, -Mir1975. -158p.

7. Varava VI (1986) Prikladnaya teoriya amortizacii transportnykh mashin - L.: Izd-vo LGU-1986. -188 p.

8. Butkovskij AG (1975) Metody upravleniya sistemami s raspredelennymi parametrami, Moscow, -Nauka, 1975. -568 p.

9. Vorobyev SA, Kostoglotov AI, Kulechov AV (2000) Mgnovenno-optimalnoe dempfirovanie kolebanyi mnogomassovych system c uprugimi svyazyami.-Izv.Vuzov, Sev-Kav. Region, Estesstvennye nauki, 2000, №2. pp. 29-32.

10. Vorobyev SA, Kostoglotov AI, Kulechov AV (2001) Sposob dempfirovaniya kolebanyi uprugopodvechennogo obyekta.. - Patent PF na izobreteniee №2162034 ot 20.01.01. 